EMERGENCY CHRONICLES 



\title{
Emergency Chronicles
}

INDIRA GANDHI AND

DEMOCRACY'S TURNING POINT

\author{
GYAN PRAKASH
}




\section{Copyright (C) 2019 by Gyan Prakash}

Requests for permission to reproduce material from this work should be sent to permissions@press.princeton.edu

Published throughout the world excluding South Asia by Princeton University Press

41 William Street, Princeton, New Jersey o8540

6 Oxford Street, Woodstock, Oxfordshire OX2O 1TR

press.princeton.edu

All Rights Reserved

Library of Congress Control Number 2018956045

ISBN 978-0-691-18672-6

British Library Cataloging-in-Publication Data is available

Editorial: Amanda Peery

Production Editorial: Ali Parrington

Jacket Design: Layla MacRory

Jacket Credit: images (clockwise) 1) Indira Gandhi / Bettmann;

2) Jayaprakash Narayan / Hulton Archive / Fox Photos;

3) advertisement from The Illustrated Weekly of India, May 1975

Production: Jacqueline Poirier

Publicity: James Schneider

This book has been composed in Arno

Printed on acid-free paper. $\infty$

Printed in the United States of America

$\begin{array}{llllllllll}10 & 9 & 8 & 7 & 6 & 5 & 4 & 3 & 2 & 1\end{array}$ 